
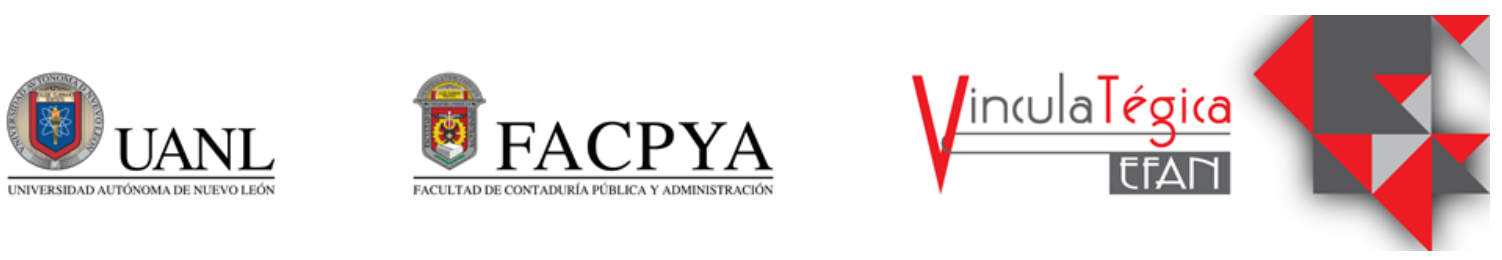

\title{
Parche localizador GPS
}

\author{
Elsa Minerva Muñoz Macías ${ }^{1}$, Juan Benito Vela Reyna ${ }^{2}$, Adelaida Figueroa Villanueva ${ }^{3}$ y Mayra \\ Yesenia Nava Rubio ${ }^{4}$
}

${ }^{1}$ Universidad Autónoma de Baja California, Facultad de Ciencias Administrativas Mexicali, Baja California México, a142274@uabc.edu.mx, Rio Nuevo y eje central s/n col. Agualeguas (+52) 686 5823377

${ }^{2}$ Universidad Autónoma de Baja California, Facultad de Ciencias Administrativas Mexicali, Baja

California México, jbenitovela@uabc.edu.mx, Rio Nuevo y eje central s/n col. Agualeguas (+52)

68658233 77Figueroa Villanueva Adelaida Rio Nuevo y eje central s/n col. Agualeguas (+52) 686

582337777 Nava Rubio Mayra Yesenia Rio Nuevo y eje central s/n col. Agualeguas (+52) 686

5823377

Información del artículo revisado por pares

Fecha de aceptación: junio-2021

Fecha de publicación en línea: diciembre-2021

DOI: https://doi.org/10.29105/vtga7.1-114

\section{INTRODUCCIÓN}

Hoy en día, en pleno siglo XXI hay varios temas de los que todavía no se puede hablar abiertamente o mencionarlo, uno de ellos es el Alzheimer. Poco a poco, nos enteramos de alguna persona que lo padece si es de un círculo cercano a nuestro alrededor. Son muy pocas las personas que lo padecen y que cuentan con el diagnostico, y que se atreven a hablar de ello o expresarlo con alguna persona a su alrededor, de la misma manera los familiares de alguna persona con este padecimiento, no se anima abiertamente a tocar el tema, se sigue manejando como tabú.

Este proyecto surge de una necesidad real, para un mercado que aún se encuentra desatendido como lo es la población de adultos mayores con Alzheimer o algun padecimiento de demencia senil, el cual apoyara a sus familiares y cuidadores, a sentirse seguros y tranquilos al momento de salir a algun lugar, que por el mismo padecimiento la persona puede extraviarse de su ser querido o de su cuidador, este proyecto ayudará a localizarlo en un menor tiempo y eso deriva a paz y tranquilidad tanto para el adulto mayor como para el cuidador, cada minuto sin poder encontrarlo es de estrés y ansiedad lo cual en un periodo largo de tiempo podría desencadenar más enfermedades.

El producto en mención es un dispositivo de localización satelital que posee varias características que lo vuelven atractivo debido su diferenciación de los demás productos de competencia, diseñado para ser utilizado en las personas de la 3er edad con alguna enfermedad como Alzheimer, o demencia senil, lo cual permita que los familiares y cuidadores de las personas con estos padecimientos puedan descansar por un momento cuando no se encuentran a su alcance de vista, y poder tener una tranquilidad mental y emocional.

El Informe mundial sobre el (Alzheimer, 2019) nos menciona que: el 75\% de las personas que están al cuidado de una persona en esta situación, se encuentran estresados entre cuidar y cumplir con otras responsabilidades y aunado a esto, también nos dice que en más del $50 \%$ de los cuidadores su salud se deterioró como resultado de sus responsabilidades. (p.13) Siendo así, una carga 
muy grande para las personas que se encuentran al cuidado de adultos mayores, por lo que se considera una oportunidad para brindar confianza y mayor tranquilidad.

Es por estas y muchas razones más, que el objetivo de este proyecto es el de brindar un dispositivo con GPS (sistema de posicionamiento satelital) para apoyar a los miembros de un núcleo familiar, con la finalidad de poder salir a pasear dar tranquilidad a la persona adulta mayor y a quienes estén a su alrededor, y si esta persona llegara a extraviarse, este sea encontrado en el menor tiempo posible, a fin de evitar todos los síntomas o traumas por los que pudiera pasar después de estar un tiempo ya sea corto o largo fuera de casa, en varios de los casos cuando se extravía una persona es por periodos largos y en pocos, ya no se vuelve a ver a la persona.

Se visualiza no solo como un producto físico que satisface alguna necesidad de geolocalización, sino como un producto que brinda la posibilidad de mayor confianza y tranquilidad a muchas personas, especialmente los adultos mayores con algun padecimiento mental, demencia senil o Alzheimer y sus cuidadores, y considerando que la esperanza de vida cada vez es mayor el mercado que existe y que existirá, será mayor. Además de que la sociedad debe de irse preparando para tener una población más longeva con todo lo que ella conlleva.

\section{MARCO TEÓRICO}

\subsection{Población en México}

Actualmente en Mexico y en el mundo, la calidad de vida de toda la población está en aumento, desde las comodidades y facilidades para vivir, para viajar, para el cuidado de la salud, comunicación, alimentación, etc. lo cual ha provocado que cada vez que más personas lleguen a vivir muchos más años de vida, y la edad promedio de la población sea aún mayor, a su vez, cada vez buscan tener menos hijos para ofrecer un mejor nivel de vida y calidad educativa, lo que nos resulta que el índice de natalidad este bajando. De acuerdo a datos del
Instituto Nacional de Geografía y Estadística por sus siglas INEGI, en su último censo poblacional del año 2020, cada vez somos más mexicanos en nuestro país. En la última década la población aumento poco más del $12 \%$, según datos referenciados del 2020, en el 2010 la población era de 112,336,538 habitantes y en el 2020 de 126,014,024 habitantes. Datos que se muestran a continuación en la siguiente tabla.

Figura 1. Población total en México según el último censo poblacional 2020 POBLACIÓN TOTAL EN MÉXICO SEGUN INEGI

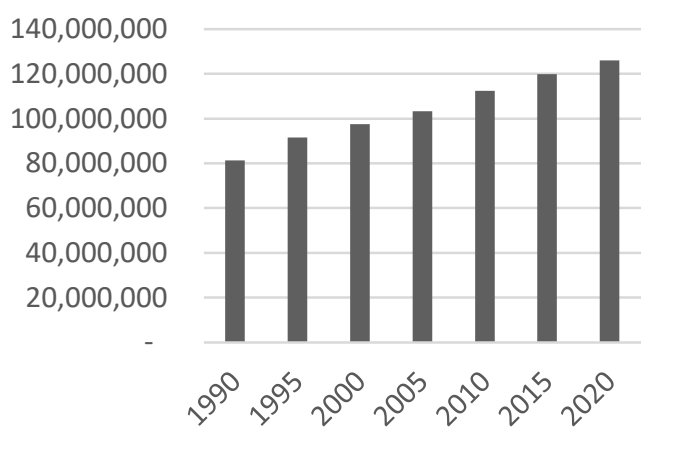

Fuente: Elaboración propia con datos de INEGI

Otro de los datos interesantes que arroja el INEGI es la cantidad de adultos mayores por cada niño o joven en la población, este dato también ha estado en crecimiento por el hecho de la calidad de vida, y que la población se está haciendo cada vez más longeva, estas personas que hace un siglo eran los pocos, ahora en el siglo XXI son los muchos, los datos que arroja a nivel Mexico son los proporcionados por en el censo intercensal INEGI 2015. El índice de envejecimiento nos refiere que, en el año 2005 por cada 100 niños y jóvenes, eran 26.4 adultos mayores, y en el 2010 por cada 100 niños y jóvenes eran 30.9 adultos mayores, estos datos para el censo intercensal del 2015 arrojaron que por cada 100 niños y jóvenes hay 38 personas de edad avanzada. Este dato no se actualizo dentro del censo poblacional del 2020. Este dato es de tomar en cuenta, ya que en una década el crecimiento de población mayor aumento un $43.93 \% 2015$ en relación con 2005. 
Figura 2. Cantidad de personas Adultas mayores por cada 100 niños y jóvenes.

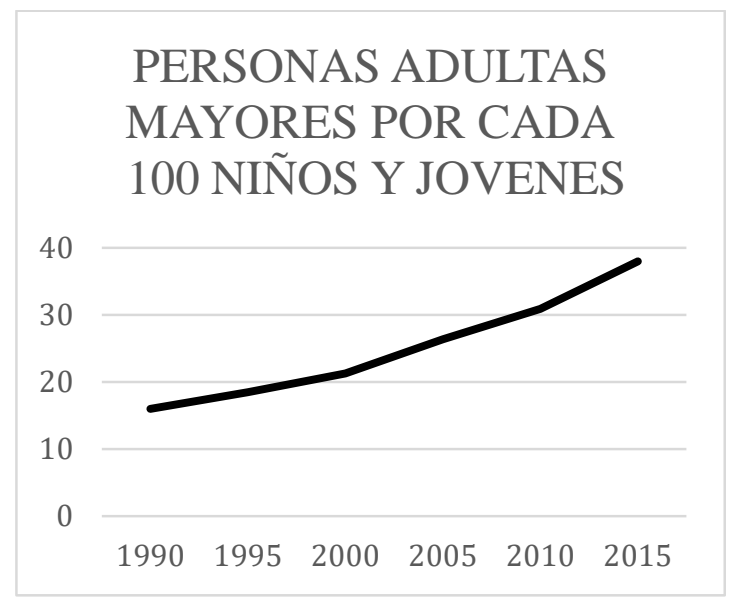

Fuente: Elaboración propia con datos de INEGI.

En un artículo dedicado al envejecimiento de la población a nivel mundial abarcando varios puntos en análisis, de longevidad, natalidad, y calidad de vida, hace referencia a la cantidad de personas a lo largo de los siglos y su expectativa de crecimiento expresada en porcentajes, nos menciona que:

La proporción de la población mundial con 65 o más años ha pasado de un 5\% a mediados del siglo XX a algo más de un $8 \%$ en el 2015; y se espera que en el 2050 llegue casi a un $16 \%$, lo cual supondrá más de 1.500 millones de personas. A finales del siglo XXI un $23 \%$ de la población y 2.500 millones de habitantes tendrán 65 o más años. (La Vanguardia, 2019, párr. 3)

Por otro lado, la siguiente tabla nos muestra datos interesantes sobre los rangos de edades de la población y en donde se concentra el grueso o promedio en nuestro país. Aquí podemos observar que el $9.12 \%$ de la población son mayores de 60 años, es decir que están dentro del rango de edades a los que nos estamos dedicando al análisis de este artículo. Dentro de este porcentaje el $4.78 \%$ son mujeres y $4.34 \%$ son hombres. Este dato se resalta por el hecho de que las mujeres son más propensas a desarrollar Alzheimer que los hombres (Alzheimer's Disease International, 2019).
También el siguiente cuadro de datos nos muestra que en cada 5 años la población se ira brincando del rango de edades, y no esta en la misma proporción de los que están naciendo, arrojando estos datos, que llegaremos a ser una población longeva como lo menciona $\mathrm{La}$ Vanguardia, 2019.

Figura 3. \% de población por edades y sexo.

\begin{tabular}{|ccc|}
\hline EDADES & MUJERES & HOMBRES \\
\hline $0-4$ & 4 & 4 \\
$5-9$ & 4.2 & 4.3 \\
$10-19$ & 8.6 & 8.7 \\
$20-29$ & 8.3 & 8 \\
$30-39$ & 7.6 & 7 \\
$40-49$ & 6.8 & 6.2 \\
$50-59$ & 5.3 & 4.8 \\
$60-69$ & 3.5 & 3.2 \\
$70-79$ & 1.18 & 1.07 \\
$80-84$ & 0.05 & 0.04 \\
85 Y MÁs & 0.05 & 0.03 \\
\hline
\end{tabular}

Fuente: Elaboración propia con datos de INEGI

Adentrando los números mencionados al estado de Baja California tenemos la siguiente informacion según el censo de población de INEGI, en el que podemos ver que el total de la población aumento un poco mas del 19\%. Y la población de adultos mayores en la federación aumento del 2020 un poco mas del $75 \%$ en relación con el 2010, y si tomamos en cuenta este dato en relación con el 2005, nos arroja un aumento del $130 \%$ en incremento de población de adulto mayor.

Figura 4. Población por edades en el estado de Baja California. 


\begin{tabular}{|c|c|c|c|c|}
\hline $\begin{array}{l}\text { Entid } \\
\text { ad } \\
\text { feder }\end{array}$ & $\begin{array}{c}\text { Grupo } \\
\text { quinquenal de } \\
\text { edad }\end{array}$ & $\begin{array}{l}2005 \\
\text { Total }\end{array}$ & $\begin{array}{l}2010 \\
\text { Total }\end{array}$ & $\begin{array}{l}2020 \\
\text { Total }\end{array}$ \\
\hline \multirow{4}{*}{$\begin{array}{l}\mathbf{B} \\
\mathbf{a} \\
\mathbf{j} \\
\mathbf{a}\end{array}$} & Total & 2844469 & 3155070 & 3769020 \\
\hline & 0 a 9 años & 54589 & 596414 & 56955 \\
\hline & 10 a 19 años & 523247 & 604667 & 627987 \\
\hline & 20 a 29 años & 488919 & 551907 & 668603 \\
\hline $\mathrm{C}$ & 30 a 39 años & 449004 & 528086 & 594458 \\
\hline $\mathbf{a}$ & 40 a 49 años & 293110 & 386153 & 534455 \\
\hline 1 & 50 a 59 años & 170980 & 234564 & 390041 \\
\hline i & 60 a 69 años & 97371 & 127789 & 228726 \\
\hline $\mathbf{f}$ & 70 a 79 años & 47738 & 61797 & 106299 \\
\hline $\begin{array}{l}\mathbf{0} \\
\mathrm{r}\end{array}$ & 80 a 89 años & 16828 & 22508 & 37655 \\
\hline n & 90 a 99 años & 2779 & 3564 & 6370 \\
\hline i & 100 años y más & 172 & 206 & 252 \\
\hline a & No especificado & 208732 & 37425 & 8219 \\
\hline
\end{tabular}

Fuente: Elaboración propia con datos de INEGI

\subsection{Alzheimer y Demencia}

Después de este análisis, podemos ver que la edad promedio de la población va en aumento, lo que, a su vez, representa que tanto México como el mundo se debe de estar preparando para los cambios y necesidades de la población. En este artículo en particular nos estaremos refiriendo a los adultos mayores, los cuales tienen derechos adicionales a los internacionales y a los que marca la Constitución Política de los Estados Unidos mexicanos. Según la OMS, menciona que dentro de los derechos universales que tenemos todos los pobladores del mundo, existen derechos adicionales para los adultos mayores los cuales hay que tomar muy en cuenta, y respecto del derecho de la salud, añade que "abarca una amplia gama de factores socioeconómicos que promueven las condiciones para que las personas puedan llevar una vida sana, y hace ese derecho extensivo a los factores determinantes básicos de la salud..." $(2015$, p.14)

Conforme el avance de los años, para toda persona es común aumentar padecimientos de salud, y para los adultos mayores la demencia es uno de los padecimientos más comunes, ya que vienen algunos de ellos por el solo transcurso del tiempo y avance de la edad. De acuerdo con la Organización de Alzheimer en la pérdida de memoria de un adulto mayor, se encuentra el Alzheimer, demencia senil y otros 11 tipos de demencia, lo cual debe ser valorado por un médico después de estudios y análisis para poderlo clasificar (Alzheimer's Association, 2016).

Ahora bien, el adulto mayor al estar padeciendo alguna de estas enfermedades, se enfrentan a discriminación de manera frecuente, por parte de las personas que no entienden la enfermedad, lo cual los lleva a ser aislados, y rezagados en alguna casa o habitación (Alzheimer's Disease International, 2019), y como consecuencia nos lleva al segundo paso, que es violar otro de sus derechos además del de la salud el de libertad, al ya no poder salir de sus casas por temor a ser rechazados por la sociedad, o por miedo a ser extraviados por consecuencia de su padecimiento de demencia o pérdida de memoria, la declaración universal de los Derechos humanos, en su articulo 3ro. Dice: "Todo individuo tiene derecho a la vida, a la libertad y a la seguridad de su persona", es por ello que con este artículo del cual realizamos este análisis, es para proveer a las personas con este padecimiento este derecho universal siempre a su alcance.

Ahora bien, el IMSS (Instituto Mexicano del Seguro Social) conceptualiza el Alzheimer como "una enfermedad que afecta las células del cerebro (neuronas), provocando que se degeneren y mueran. Quienes la padecen presentan un deterioro progresivo en la capacidad para procesar el pensamiento (Memoria, orientación, lenguaje, aprendizaje, cálculo, etc)." (IMSS, s.f.) este padecimiento ya se encuentra tratado en Mexico, lo cual es un avance, pero el crecimiento de la población va mucho más rápido que lo que se está preparando el país para esto.

El concepto de demencia para (Gutierrez L.M., García M.C., Roa P.A., Martínez A., 2017) es el siguiente "es un síndrome de etiología diversa, que afecta progresivamente la memoria, el pensamiento y la habilidad para realizar actividades de la vida cotidiana" (p. 24), este concepto explica de una manera breve y clara el proceso de la enfermedad $y$ el porque estas personas necesitan de un apoyo para su tranquilidad, tanto ellos como sus cuidadores, que es el nombre que se les da a las personas que están 
a cargo de alguna persona adulta mayor, los cuales también presentan algun tipo de trastorno en el cual según la $\mathrm{ADI}$ en una encuesta aplicada a nivel mundial destaca que en más del $50 \%$ su salud se deterioro por causa de la responsabilidad que el cuidado del adulto mayor con este tipo de padecimiento le implica. (ADI, 2019, p.6) todo esto ha llevado a voltear y analizar detenidamente también hoy en día al cuidador, el ver por su salud física, mental y emocional.

Por todas las razones anteriores expuestas es que dentro de este artículo mencionamos el uso de un parche GPS para que el adulto tenga un envejecimiento saludable y seguro, el cual analizaremos y detallaremos más adelante. Por lo pronto veremos el cómo funciona y ventajas que ofrece este dispositivo.

\subsection{GPS. Sistema de Posicionamiento Global}

El concepto oficial de GPS, de acuerdo con Estados Unidos de Norteamérica es "El Sistema de Posicionamiento Global (GPS) es un sistema de radionavegación de los Estados Unidos de América, basado en el espacio, que proporciona servicios fiables de posicionamiento, navegación, y cronometría gratuita e ininterrumpidamente a usuarios civiles en todo el mundo.” (GPS.GOV, s.f.)

Este sistema de posicionamiento su generalidad es el de ubicar en tiempo y espacio al objeto en cuestión, en este caso lo estaremos aplicando para el objetivo de este artículo en personas, especialmente aplicadas en personas de la tercera edad con algun padecimiento de demencia senil o Alzheimer.

Hoy en día, existe GPS casi en cualquier celular, o algun dispositivo electrónico que el adulto mayor pudiera llegar a utilizar, pero en nuestro particular estudio, se ve que las personas con padecimientos de demencia senil o Alzheimer tienden a olvidad objetos en cualquier lugar sin darse cuenta, ni recordarlo y nuestro objetivo es el de encontrar al adulto mayor en el menor tiempo posible para que no ocasione ningún tipo de estrés ya sea a la persona extraviada o a su cuidador. Por lo que la propuesta de este estudio es proporcionar un GPS en un tipo de presentación práctico y fácil de usar y portar para el adulto.

\section{MÉTODO}

\subsection{Motivo por el que es importante el crear un parche GPS.}

Se desea crear una forma de posicionar a nuestros adultos mayores, en especial a los que tengan algun padecimiento de Alzheimer o demencia. Todo esto con la finalidad de ofrecer la tranquilidad de seguridad al adulto mayor, así como a su cuidador, poder ofrecerle el sentirse libre y poder salir a la calle, sin necesidad de ser observado el $100 \%$ del tiempo que se encuentre fuera de su lugar habitual de vivienda.

\subsection{Productos Sustitutivos.}

Actualmente con el avance de la tecnología, existen varios artículos que poseen GPS, los cuales varían desde un dispositivo pequeño portátil, hasta el celular que hoy en día, la mayoría de la población cuenta con uno.

Existen varios rastreadores portátiles, de tamaño mínimo desde medidas de $3 \mathrm{~cm} \times 3 \mathrm{~cm}$ $\mathrm{x} 1 \mathrm{~cm}$. Los cuales presionas un botón al momento de sentirte inseguro y contiene una pila de larga duración única. También existe el reloj inteligente, el cual incluye GPS, y muestra además de la hora y mediciones de salud al adulto muy práctico y fácil de portar. También existe otro tipo de rastreador el cual es portátil y fácil de colocar dentro de las prendas del adulto mayor, que manda solamente señal de donde se encuentra y su pila es de duración de 25 días.

\subsection{Parche localizador GPS.}

¿Porque inclinarnos hacia el parche GPS? Porque es importante el tomar como articulo preferente el parche GPS, ¿para el adulto 
mayor? ¿Y porque en especial para adultos con algun padecimiento de demencia senil o Alzheimer? Los adultos mayores con este tipo de padecimiento al extraviarse, olvidan todos los artículos que traen consigo, es por eso que los celulares dejan de ser el producto más atractivo y fácil para rastrear, aunado a esto estas personas al verse en calidad de desorientadas tienden a ser presa fácil para un asalto, en el que por lo regular las prendas de vestir, celulares, relojes, se vuelven los artículos mas atractivos para el robo, dejando atrás al reloj inteligente y varios rastreadores portátiles.

Es por ello que, el estudio y análisis de este articulo es el de ofertar a esta población un articulo que pueda ser cómodo de portar, sea funcional y práctico y con un sistema interactivo para ser utilizado por los familiares y cuidadores sin complicaciones, además de ser agradable a través de la aplicación, pero altamente funcional, optimizado para lograr una localización en tiempo real y lo más exacta posible del dispositivo.

\section{RESULTADOS}

El resultado de nuestra investigación nos arroja que se necesita ofrecer tanto al adulto mayor con alguna enfermedad de demencia senil o Alzheimer la tranquilidad de poder salir y sentirse con esa libertad de poder pasear por algun momento sin la sensación de extraviarse, ya sea de la persona o de su cuidador, actualmente existen varios dispositivos que cuentan con GPS, iniciando con el propio celular, pero este se convierte en un objeto que puede ser fácilmente olvidado en cualquier parte por una persona con este tipo de padecimientos. Es por ello que la forma propuesta de parche es la ideal, para poder ofrecer al adulto mayor comodidad al utilizarlo, además de que está siendo creado especialmente para adultos mayores. $\mathrm{Al}$ ofrecer un parche GPS, este sería adherido en su cuerpo en forma de curita, para su comodidad y no olvidarlo en ningún sitio y así lograr el objetivo de ser localizado el $100 \%$ del tiempo que se encuentre fuera de su habitual vivienda.
Tomando en cuenta el avance de la tecnología y el que hoy en día sin importar la situación es importante el vernos bien, se ofrecería el parche en 3 colores, las cuales serían para alinearse a los 3 tonos de piel que prevalecen en el mundo, principalmente en México y Estados unidos, tomando en cuenta estos países como principales lugares para el arranque del producto. Este parche seria en forma de curita, con un diseño cuadrado y en forma de bolsa, todo esto con la finalidad de colocar el GPS dentro del parche. El parche seria creado de tal manera que tendría que ser desechable para poderlo utilizar únicamente en las ocasiones en que el adulto mayor con este padecimiento salga a la calle, para no estar en contacto con su piel todo el tiempo. Tomando en cuenta que para un adulto mayor su dermis tiende a tener resequedad, este parche tendría aloe, dentro del pegamento para no causar algun daño temporal o permanente en su piel.

Este GPS se activaría directamente con 2 teléfonos celulares para su ubicación el 100\% del tiempo, contaría una pila la cual se cargaría de manera táctil, y su duración seria de más de 48 horas, dicho GPS se introduce al momento de utilizarlo al parche. Para contar con este servicio se estaría pagando una mensualidad, la cual sería una renta mínima, al contar únicamente con el servicio de GPS.

El parche GPS es un producto que posee varias cualidades diferenciadoras de lo que actualmente existe en el mercado, este se caracteriza por ser funcional, práctico, ligero e imperceptible convirtiéndolo en un producto seguro y privado.

Es funcional y práctico porque requiere de un sistema interactivo sencillo que puede ser utilizado por los familiares y cuidadores sin complicaciones, además de ser agradable a través de la aplicación, pero altamente funcional, optimizado para lograr una localización en tiempo real y lo más exacta posible del dispositivo.

Seguro, debido a que se puede poner en cualquier parte del cuerpo para que no sea visible, y al ser ligero y pequeño es tan 
cómodo que la persona no sentirá incomodidad.

\section{CONCLUSIONES}

En este siglo XXI que de manera consciente y científica nos está mostrando que la población va en aumento rápidamente, y que la tecnología y la ciencia están avanzando a pasos todavía más veloces que la población, no podemos cerrar los ojos y enfocarnos en crear productos y artículos solo para la infancia, juventud, y solamente ofrecer al consumidor lo que necesita en su etapa productiva.

Debemos de empezar a ser emprendedores creativos y pensar fuera de la caja, creando artículos para la población en la que en algun momento estará la mayoría. Actualmente los adultos en edad productiva se preocupan más por prever el cómo vivirán al momento de su retiro, y preparan un retiro tranquilo, saludable y con comodidades. Tomando en cuenta que según las estadísticas es un gran porcentaje de los adultos mayores que en algun momento de su edad desarrollaran algun tipo de demencia y en otros hasta Alzheimer.

es por ello que debemos de pensar en que este producto de Parche GPS, viene a dar libertad a las personas adultas mayores para poder pasear y salir a la calle sin temor a ser extraviados. El miedo existe, en la persona que en algun momento se encontrara en esa situación, así como en los familiares de los que ya lo padecen y de los cuidadores, que, por temor de extraviar al familiar o amigo, los mantienen en algun lugar encerrados perdiendo uno de los derechos universales que es la libertad, y otro que es el de la salud, incluyendo la salud mental tanto de la persona que lo padece como del cuidador.

Finalmente, este parche viene a ofrecer una tranquilidad de pasear con tranquilidad y seguridad. 


\section{REFERENCIAS}

Alzheimer's Association. (2016). Información Básica Sobre la Enfermedad de Alzheimer.

Alzheimer's Disease International. (2019). Informe Mundial sobre el Alzheimer 2019: Actitudes hacia la demencia

Censo poblacional Inegi,

GPS.GOV (s.f), Consultado el 03 de abril del 2021. https://www.gps.gov/spanish.php\#: :text=El\%20Sistema\%20de\%20Posicionamiento\%20G1 obal\%20(GPS)\%20es\%20un\%20sistema\%20de,civiles\%20en\%20todo\%20el\%20mundo.

Gutierrez L.M., García M. C., Roa, P. A., Martínez A., Eds. Conacyt (2017), La enfermedad de Alzheimer y otras demencias como problema nacional de salud, Documento de postura, 1era edición, SBN 978-607-443-721-8, https://www.anmm.org.mx/publicaciones/ultimas_publicaciones/ANM-ALZHEIMER.pdf

IMSS. (s.f.) Enfermedad de Alzheimer. Consultado el 03 de abril de 2021. http://www.imss.gob.mx/salud-en-linea/enfermedad-alzheimer

La Vanguardia (17 de diciembre de 2019), "Así transformará nuestro mundo el envejecimiento de la población”, $\quad h$ ttps://www.lavanguardia.com/vanguardiadossier/20191216/472203714080/demografia-cambiar-mundosociedad.html\#: :text=La\%20proporci\%C3\%B3n\%20de\%20la\%20poblaci\%C3\%B3n,de\%2 01.500\%20millones\%20de\%20personas. Recuperado el 16 de abril de 2021 de https://www.lavanguardia.com/vanguardia-dossier/20191216/472203714080/demografiacambiar-mundosociedad.html\#: :text=La\%20proporci\%C3\%B3n\%20de\%20la\%20poblaci\%C3\%B3n,de\%2 $01.500 \% 20$ millones\%20de\%20personas.

Naciones Unidas (s.f), "La Declaración Universal de los Derechos Humanos", https://www.un.org/es/about-us/universal-declaration-of-human-rights.

Organización Mundial de la Salud (2015), Informe Mundial sobre el envejecimiento y la salud, ISBN $\begin{array}{lllllll}978 & 92 & 4 & 069487 & 3 & \text { (PDF), recuperado }\end{array}$ http://apps.who.int/iris/bitstream/handle/10665/186466/9789240694873_spa.pdf;jsessionid= 38A9DD6B616A3E2AA8EE4C4B132196B2?sequence=1 